\title{
Design and implementation of furnace temperature measurement system for power plant coal-fired boiler in acoustic method
}

\author{
Feng Tian ${ }^{1}$, Hongguang Zhang ${ }^{1, \mathrm{a}}$ and Yan Tian $^{2}$ \\ ${ }^{1}$ School of Automation, Shenyang Aerospace University, Shenyang 110136, China \\ ${ }^{2}$ Beijing Tiandi Make Power Industry Control System Co., Ltd, Beijing 100013, China
}

\begin{abstract}
The furnace temperature of coal-fired boilers in power plants is an important operating parameter for boiler flame monitoring and control, which directly affects the combustion efficiency and the safety of boilers. Based on the research status at home and abroad and the previous research foundation, this paper studies and designs the temperature measurement system of acoustics method. The principle of temperature measurement by sound method is discussed in this paper, and the generalized cross correlation (GCC) method for measuring flight time of sound waves is introduced. The software and hardware of the temperature measurement system are designed in detail. The experiment is carried out according to the measured data of power plant and the results show that the system can measure the average temperature of acoustic paths in the furnace. The feasibility of the system is verified. And this study provides a basis for the reconstruction of furnace temperature field.
\end{abstract}

\section{Introduction}

The temperature in the coal-fired boiler of a power plant is an important physical quantity reflecting the combustion efficiency, boiler safety and other indicators [1]. Through to the furnace internal temperature field measurement, the staff can timely adjust the boiler running state, achieve the optimal control of coal-fired boiler, so it is particularly important to real-time measure the temperature.Acoustic temperature measurement is a mainstream high temperature measurement method at present. It has the characteristics of non-contact, high efficiency and high precision.

Based on ARM chips controller, by controlling the acoustic transceiver working state, the system uses one A/D converter to acquire acoustic sensor signal and processes the signal through the generalized cross correlation method to get the travel time of the sound wave on the path. Then the average temperature on the path can be obtained, which is based on the function relation between the propagation speed of sound wave and the temperature of the medium.

\section{System working principle}

The principle of acoustic temperature measurement technology is based on the function relation between the velocity of sound wave propagation and the temperature of medium, and also related to the composition of gas in furnace. From a theoretical perspective, the deviece required for the acoustic temperature measurement technology is not complicated. Several acoustic wave transmitters and acoustic wave receivers can be installed around the furnace to be tested, and by measuring the propagation time for the acoustic wave to reach the receiver from the transmitter [2]. The sound wave propagation speed and the average temperature on the corresponding path can be calculated. Then the temperature field distribution in the furnace can be restored according to the temperature field reconstruction algorithm. The function between the propagation speed of sound waves inside the furnace chamber and the gas composition can be expressed as:

$$
c=\sqrt{\frac{\gamma \cdot R}{M} T}=Z \sqrt{T}
$$

Where: $c$ is the speed of sound wave propagation in the medium, $\mathrm{m} / \mathrm{s} ; \gamma$ is the adiabatic index of furnace flue gas; $R$ represents the gas constant, $\mathrm{kJ} /(\mathrm{mol} \cdot \mathrm{K}) ; M$ is the molar mass of the gas, $\mathrm{g} / \mathrm{mol} ; T$ is the absolute temperature of the medium, $\mathrm{K} ; Z=\sqrt{\gamma \cdot R / M}$.

In a specific furnace environment, $\gamma, R, M$ change very little, which are considered as constant, so the speed of sound waves inside the furnace depends mainly on the temperature of the medium.In practical engineering applications, two sets of acoustic wave transmitters and acoustic wave sensors are installed on each of the walls of the furnace.

The detailed working process of acoustic temperature measurement system is as follows: The controller controls the acoustic wave transmitter in different positions to sound in sequence. When one sound wave transmitter works, the acoustic wave sensor converts the acoustic signals into analog electrical signals, and outputs them to the $\mathrm{A} / \mathrm{D}$ converter inputs through

* Corresponding author:a Hongguang Zhang: zhg1023058880@163.com 
amplifiers and filters. The controller controls the A/D converter to acquire signals from the two acoustic sensors on the path. The generalized cross-correlation method is applied to process the signals, and the position of the peak of the cross-correlation function is obtained. The system combines the sampling frequency and the peak position of the cross-correlation function to calculate the flight time of the acoustic wave on the path, and then obtains the acoustic wave velocity and the average path temperature.

The correlation function method is widely used in the field of signal detection, such as detection and extraction of signals in noise, implicit periodic detection in signals, detection of signal correlation, and measurement of signal delay time [3]. In addition, the correlation function is an important statistic describing the random signal, and the correlation function of the random signal and its power spectral density are a pair of Fourier transform pairs. In the acoustic temperature measurement system, the measurement of the acoustic flight time is crucial. The measurement accuracy will directly affect the accuracy of the system temperature measurement [4]. Taking a single path as an example, the mathematical model of the acoustic signal is:

$$
\left\{\begin{array}{l}
x_{1}(n)=s(n)+\omega_{1}(n) \\
x_{2}(n)=\alpha s(n-\tau)+\omega_{2}(n)
\end{array}\right.
$$

Where: $x_{1}(n)$ is the discrete signal of the acoustic sensor adjacent to the acoustic transmitter in operation; $x_{2}(n)$ is the discrete signal of the acoustic sensor on the other side of the path; $s(n)$ is the signal of the sound wave transmitter; $\omega_{1}(n)$ and $\omega_{2}(n)$ are the noise signals received by the corresponding acoustic sensors; $\alpha$ is sound attenuation coefficient; $\tau$ is the delay time between two acoustic sensor signals.

According to some statistical studies, the correlation function of $x_{1}(n)$ and $x_{2}(n)$ can be expressed as:

$$
R_{12}(t)=E\left[x_{1}(n) x_{2}(n+t)\right]
$$

Where: $E[\cdot]$ is the mathematical expectation.

By using Eqs. (2), (3), it is obtained that

$$
\begin{aligned}
R_{12}(t)= & \alpha E[s(n) s(n-\tau+t)]+E\left[s(n) \omega_{2}(n+t)\right]+ \\
& \alpha E\left[s(n-\tau+t) \omega_{1}(n)\right]+E\left[\omega_{1}(n) \omega_{2}(n+t)\right]
\end{aligned}
$$

The sound source signal $s(n)$, noise signal $\omega_{1}(n)$, and $\omega_{2}(n)$ are not correlative [5], so it is obtained that:

$$
\left\{\begin{array}{l}
E\left[s(n) \omega_{2}(n+\tau)\right]=0 \\
E\left[s(n-\tau+t) \omega_{2}(n)\right]=0 \\
E\left[\omega_{1}(n) \omega_{2}(n+\tau)\right]=0 \\
R_{12}(t)=\alpha E[s(n) s(n-\tau+t)]=\alpha R_{s}(t-\tau)
\end{array}\right.
$$

Where: $R_{s}$ is the autocorrelation function of $s(n)$.

According to the nature of the correlation function, when $t=\tau, R_{s}(t-\tau)$ reaches a maximum value, that is,
$R_{12}(t)$ reaches the maximum value, so when the maximum value of $R_{12}(t)$ is obtained, and the delay time of the two acoustic wave sensor signals can be obtained. According to Wiener-Khintchine theorem, it is obtained that:

$$
\left\{\begin{array}{l}
G_{12}(k)=\overline{X_{1}(k)} X_{2}(k) \\
R_{12}(t)=I D F T\left[G_{12}(k)\right]
\end{array}\right.
$$

Where: $G_{12}(k)$ is the cross-power spectrumdensity function of $x_{1}(n)$ and $x_{2}(n) ; D F T[\cdot]$ is the discrete Fourier transform, and IDFT [.] represents the inverse discrete Fourier transform; $\overline{X_{1}(k)}$ indicates the conjugate of $\quad X_{1}(k) \quad ; \quad X_{1}(k)=D F T\left[x_{1}(n)\right]$, $X_{2}(k)=D F T\left[x_{2}(n)\right]$.

The system applies the generalized cross-correlation method (GCC) to process the collected data according to the information of background noise in the boiler, and weights the cross-power spectrumdensity function to suppress the effect of noise and sharpens the peak of the cross-correlation function [6]. In addition, when the amount of processed data is large, the amount of calculation is large by performing the discrete Fourier transform. Therefore, it is impractical to directly use DFT for real-time processing of signals. Considering the above two factors, the system adopts the generalized cross-correlation method based on phase transform (PHAT-GCC), which has a certain anti-reverberation effect. And Fast Fourier Transform (FFT) is used to process signals, which improves the efficiency of the system greatly. It can be expressed as:

$$
\left\{\begin{array}{l}
X_{1}(k)=F F T\left[x_{1}(n)\right] \\
X_{2}(k)=F F T\left[x_{2}(n)\right] \\
R_{12}(t)=\operatorname{IFFT}\left[\overline{X_{1}(k)} X_{2}(k) H(k)\right]
\end{array}\right.
$$

Where: $H(k)=1 /\left|G_{12}(k)\right|$ is PHAT weighting function.

The controller calls the DSP instruction to obtain the position of the correlation function peak. The function between the flight time of sound waves and the path average temperature can be expressed as:

$$
\left\{\begin{array}{l}
\tau=\frac{k}{F_{S}} \\
T=\left(\frac{d}{\tau \cdot Z}\right)^{2} \times 10^{6}-273.16
\end{array}\right.
$$

Where: $\tau$ is the flight time of sound waves on the path, $\mathrm{ms} ; k$ is the position of the cross-correlation function peak; $F S$ is sampling frequency, $\mathrm{kHz}$; $d$ is the length of the sound propagation path, that is, the distance between the acoustic wave sensors on one path, $\mathrm{m} ; T$ is the average temperature of the sound wave propagation path, ${ }^{\circ} \mathrm{C}$.

\section{System composition and hardware implementation}


The system consists of an industrial computer, one master control board, the signal conditioning circuits, sound wave generators and acoustic wave sensors. The master control board includes ARM controller module, one A/D conversion module, an acoustic wave transmitter switching module and a communication module. The system composition and hardware structure diagram is shown in Figure 1. The path number and the reference temperature of each path are shown in the figure.

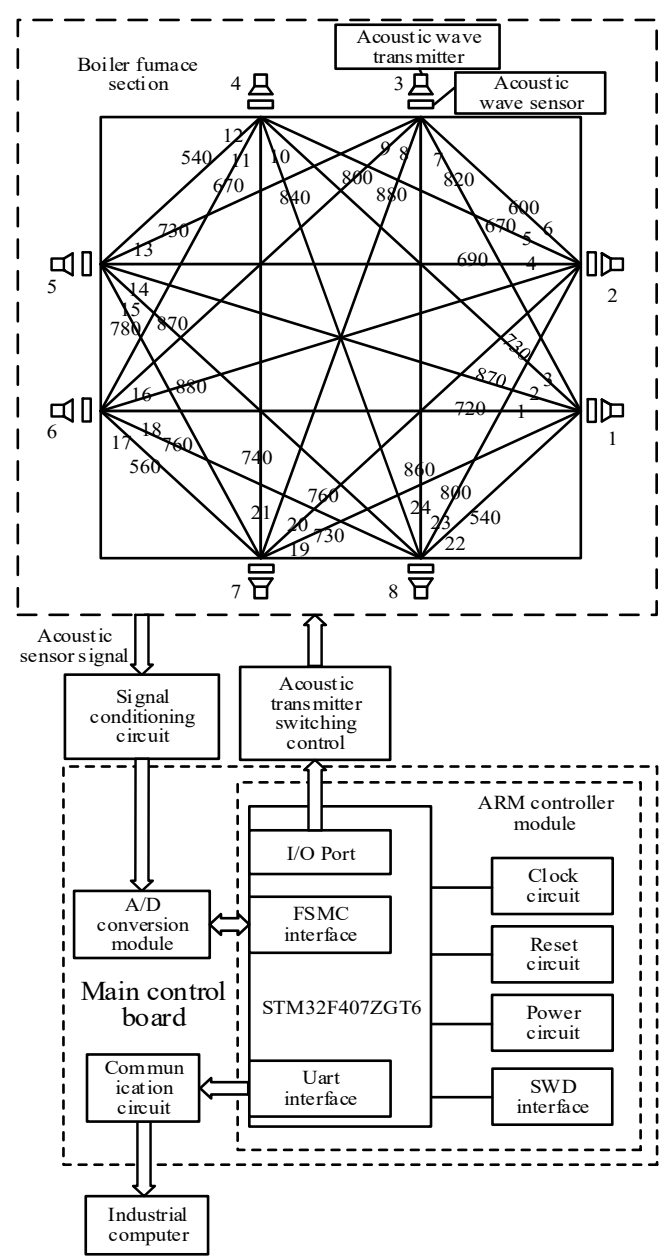

Figure 1. System composition and hardware structure diagram

\subsection{ARM controller module}

ARM controller module is composed of ARM chip, power supply circuit, reset circuit, clock circuit and SWD circuit. The controller module selects the 32-bit STM32F407ZGT6 chip of ST (STMicroelectronics) as the master control chip, which has rich resources, powerful function. The MCU is a CortexTM-M4 core and incorporates FPU (floating-point arithmetic) units and DSP instructions inside it. It has $1 \mathrm{MB}$ of Flash memory. The clock circuit uses an external $8 \mathrm{M}$ crystal oscillator, which can reach up to $168 \mathrm{MHz}$ through the frequency multiplication by the MCU's internal phaselocked loop. This allows the MCU to have high-speed computing and processing capabilities. The reset circuit adopts the combination of power-on reset and key-reset reset, which is simple and convenient. The SWD circuit is designed for program downloading and debugging. STM32F407ZGT6 has rich peripheral resources and provides strong support for the design and development of the system. In the system design process, the chip's FSMC (flexible static memory controller) interface is fully utilized.

\subsection{Signal conditioning circuit}

When the system acquires the acoustic wave sensor signal, the output signal of the sensor is weak and contains noise interference. To improve the data acquisition accuracy, the sensor output signal should be amplified and meet the input range requirements of the A/D conversion chip. In addition, the amplified signals need to be filtered in order to remove noise interference [7].

\subsubsection{Signal amplifier circuit}

The output signal range of the acoustic wave sensor is $50 \mathrm{mV}$ to $+50 \mathrm{mV}$, while the analog input signal range of the AD7606 is $\pm 5 \mathrm{~V}$ or $\pm 10 \mathrm{~V}$. Therefore, it is necessary to design a signal amplifying circuit to convert the $50 \mathrm{mV}$ to $+50 \mathrm{mV}$ signal into the $-5 \mathrm{~V}$ to $+5 \mathrm{~V}$ signal. The design uses a high-precision, low-power, low-noise railto-rail instrumentation amplifier, the AD8422, to amplify the sensor output signal. The device processes the signal with ultra-low distortion performance, and the load does not affect performance over the output range. The amplifier only needs to connect an external resistor between the two $R_{G}$ pins to complete the gain configuration. The amplification gain can be expressed as:

$$
G=1+\frac{19.8 \mathrm{k} \Omega}{R_{G}}
$$

Where: $G$ is the gain multiple; $R_{G}$ is the external resistor, $\mathrm{k} \Omega$.

According to the requirement, the amplifier gain needs to be set to 100 . To accurately adjust the gain of the amplifier, the external resistor uses a precision resistor of $200 \Omega$. The output signal of the AD8422 is connected to the input of the filter circuit. The amplifier circuit is shown in Figure 2.

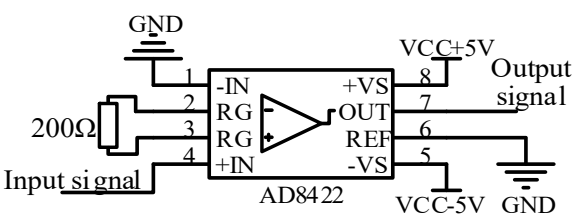

Figure 2. Signal amplifier circuit

\subsubsection{Signal filter circuit}

During the operation of the system, there is background noise inside the furnace. According to experimental research, the noise is mostly low frequency noise, which is concentrated below $400 \mathrm{~Hz}$. On the other hand, in the 
acoustic signal generated by the acoustic wave transmitter, the higher frequency part will have a great attenuation in the process of propagation, which seriously affects the consistency of output signals of the acoustic wave sensors. Therefore, before the sound wave signal is sampled, it is necessary to perform filtering processing on the signal after the amplification circuit.

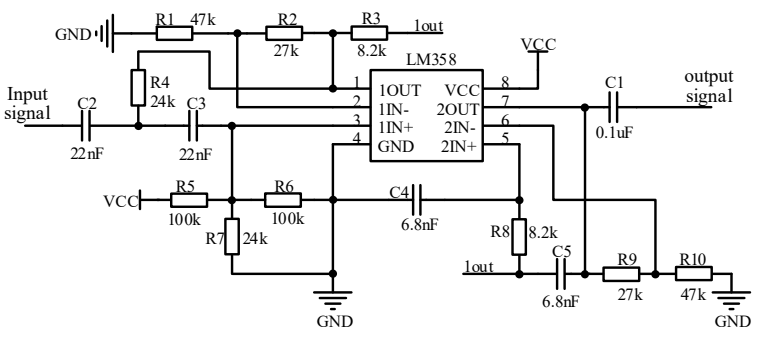

Figure 3. Filter circuit

According to the requirements, the system uses a general-purpose operational amplifier LM358 to design an active bandpass filter with a passband of $400 \mathrm{~Hz}$ to $2000 \mathrm{~Hz}$. The circuit is shown in Figure 3, where the first stage is a high-pass filter and the second stage is a lowpass filter. The output signal of the filter circuit is connected to the input of the A/D conversion module. The cutoff frequency is expressed as:

$$
f=\frac{1}{2 \pi R C}
$$

Where: for the high pass filter, $C=C_{2}=C_{3}, \mathrm{nF}$; $R=R_{4}=R_{7}, \mathrm{k} \Omega$; for the low pass filters, $C=C_{4}=C_{5}$, $\mathrm{nF} ; R=R_{3}=R_{8}, \mathrm{k} \Omega$.

\subsection{A/D conversion module}

The system needs to acquire the signal of the acoustic wave sensors in real time and accurately, but the resolution of the A/D converter inside the STM32 is 12bit, and the accuracy of the $\mathrm{A} / \mathrm{D}$ converter cannot meet the system requirements. Therefore, the A/D conversion module is expanded outside the system, and the AD7606 chip is selected as the A/D conversion chip, which is a 16-bit, 8-channel analog-to-digital data acquisition chip and contains analog input clamp protection, a secondorder antialiasing filter, a track-and-hold amplifier, a flexible digital filter, a $2.5 \mathrm{~V}$ reference and reference buffer, and high speed serial and parallel interfaces. The AD7606 is powered by a single 5V supply and can accommodate $\pm 10 \mathrm{~V}$ and $\pm 5 \mathrm{~V}$ true bipolar input signals while sampling at a throughput rate up to $200 \mathrm{kSPS}$ for all channels, which can meet the performance specifications of this system design.

In the system, the parallel interface of the AD7606 is used to ensure fast signal acquisition. The system makes full use of the STM32 FSMC (Flexible Static Storage Controller) interface, and the AD7606 is connected to STM32 through the parallel interfaces. In this way, the STM32 accesses the AD7606 chip as an external storage and AD7606 is equivalent to an external memory with only one address, which can achieve fast and convenient control and access to the AD7606. The data lines FSMC D0 to FSMC D15 of the FSMC interface are connected to the parallel data lines DB0 to DB15 of the AD7606 in sequence. FSMC NE1 is connected as a chip selection signal to the CS pin of the AD7606. STM32 outputs a PWM signal to the CONVST A, B pins of the AD7606 through an internal timer, which is used to control the data sampling frequency. FSMC NOE is connected to the pin of the AD7606 as a reading time sequence. The BUSY pin of the AD7606 acts as an external interrupt input of the STM32 to detect the transition state. When CONVST A, B pins reach the rising edge, the BUSY pin becomes a logical high level, indicating the beginning of the conversion process. During this process, the BUSY pin maintains a high level. When the conversion of all channels is completed, the pin changes from a logical high level to a low level, at which point the conversion result can be read in the interrupt function. The RANGE pin is tied to GND, and the voltage input range is set to $\pm 5 \mathrm{~V}$ for all channels. The OS2, OS1, and OS0 pins are used to set the oversampling ratio, but the system does not use the oversampling function. The RESET pin is used to complete the reset operation of the AD7606. The interface circuit of AD7606 and STM32 is shown in Figure 4.

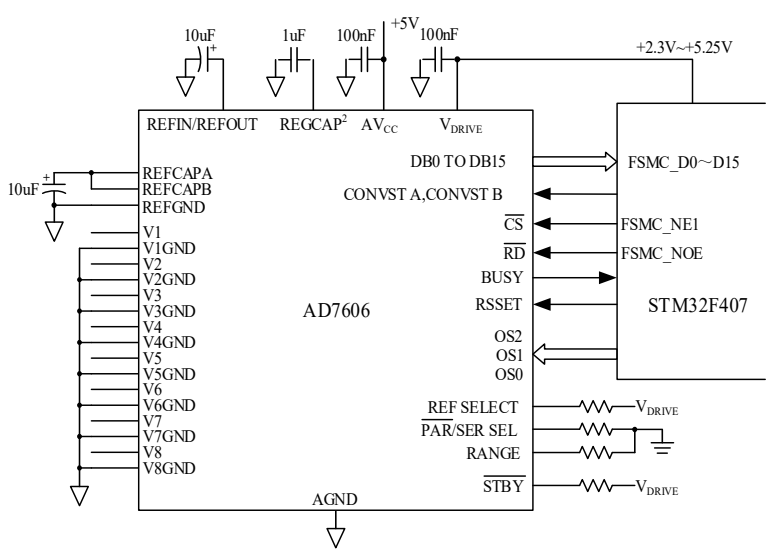

Figure 4. AD7606 interface circuit

The AD7606 outputs the conversion results in binary complement. It can be expressed as:

$$
C O D E=\frac{V i n}{5 \mathrm{~V}} \times 32768 \times \frac{R \mathrm{e} f}{2.5 \mathrm{~V}}
$$

Where: $C O D E$ is the encoding result of $\mathrm{AD} 7606$ conversion; Vin is the analog signal input voltage of the AD7606, V; Ref is the AD7606 reference voltage,internal reference voltage of $2.5 \mathrm{~V}$ is selected in the system, $\mathrm{V}$.

\subsection{Acoustic transmitter switch module}

According to the principle of acoustic temperature measurement, only one acoustic wave transmitter generates sound waves when the system is running. To complete the temperature measurement of all paths, it is necessary to design the acoustic transmitter switch circuit, and the acoustic wave transmitter is driven by a 
solenoid valve. So the system uses a 8-channel analog multiplexing switch CD4051B, optocouplers and Darlington Transistor Arrays ULN2803 to design the switching circuit. The circuit structure is shown in Figure 5 , and only a single optocoupler is shown.

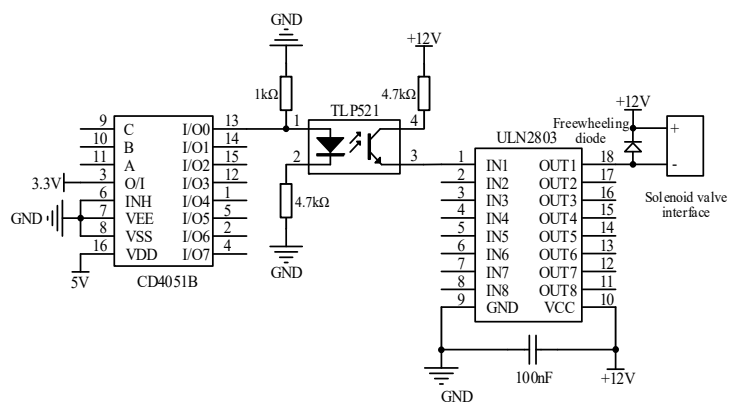

Figure 5. Acoustic transmitter switching module

The pins A, B, and C of the CD4051B are connected to the general-purpose IO port of the controller to control the gate of the CD4051B. When A, B, and C are logic low, pin $13, \mathrm{I} / \mathrm{O} 0$ is connected to the common pin 3, I/O0 outputs a high level to the input of the optocoupler. The optocoupler is turned on and outputs a high level to the IN1 input of the ULN2803. Because ULN2803 is an open collector output mode, OUT1 outputs a low level. The solenoid valve is turned on, and the acoustic transmitter emits sound waves.

\subsection{Communication module}

The ARM controller communicates with the industrial computer through the serial port. The system adopts RS85 interface, which is widely used in industrial communication field. It has the characteristics of high transmission rate, strong anti-common-mode interference capability and long transmission distance. It has been one of the mainstream serial communication interfaces. The slave machine datas are transmitted through the RS-485 interface, and the RS485 level signal is converted into a USB interface signal to the computer by using an RS485/USB converter. This connection method makes full use of the characteristics of the USB interface, greatly improving the versatility and transmission rate of the design.

\section{System software implementation}

For the purpose that the average temperature on the path is measured, the system needs to simultaneously acquire the acoustic wave sensor signals at the sound wave transmitting end and the sound wave receiving end [8]. The system uses the generalized cross-correlation algorithm to calculate the peak position of the crosscorrelation function, combining the sampling frequency and path length to acquire the average temperature on the path. The controller controls switching of the acoustic wave transmitters, traversing all the paths, completes the temperature measurement. The system upload data to the computer through the serial communication module. The system software mainly includes system initialization, acoustic transmitter switching module, data acquisition module, data processing module and the communication module. The system initialization is the entry of the main program, and completes the preparation of each module. The system software process is shown in Figure 6.

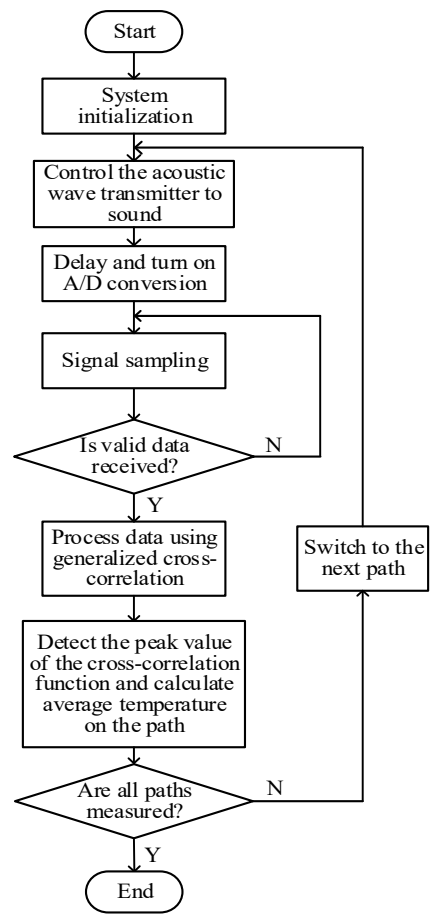

Figure 6. Main program flow chart

First, the program initializes the system. In the initialization phase, it mainly completes the configuration of the system clock and various peripherals, and enable the FPU (floating point arithmetic unit) and DSP instructions. The clock configuration is mainly the STM32 chip clock initialization. Peripheral configuration includes GPIO port configuration, the timer initialization, FSMC interface initialization, and serial port initialization, etc.

According to the introduction of the A/D conversion module, during the initialization of the FSMC interface, the system configures the first area of Bank 1 of the FSMC. In data acquisition module, the controller read the datas from the slave device AD7606 by FSMC interface. The address of the AD7606 is 0X60000000. In a single acquisition process, the controller can only obtain the data of one sampling point of each channel. In order to meet the requirements of the generalized crosscorrelation algorithm for data volume, when the system measures the average temperature of the path, multipoint sampling of the output signal of the acoustic wave sensor is performed to get enough data.

\section{Experimental results and analysis}

In summary, the acoustic temperature measurement system was designed, and the system was verified by combining the measured data of the power plant. The sampling frequency of the configuration system is 16 $\mathrm{kHz}$, and the number of sampling points of each channel is 1024 . The sampling data is calculated by the PHATGCC method, and the cross-correlation function is 
shown in Figure 7. It is concluded from the image that the PHAT-GCC method can obtain the cross-correlation function well, and the peak of the correlation function is easy to extract.

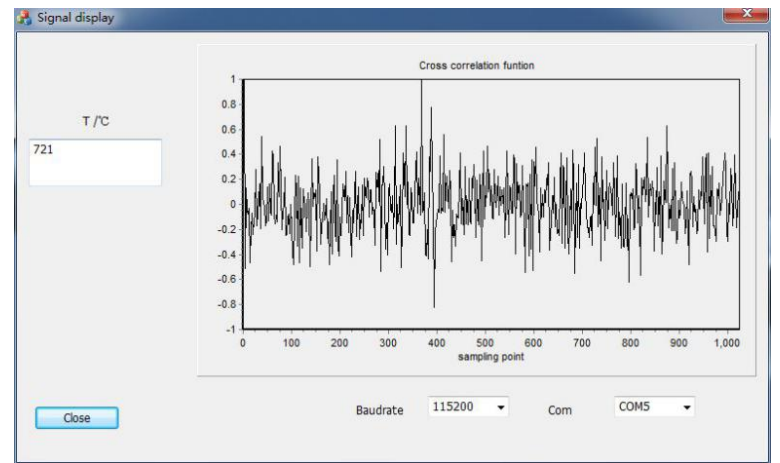

Figure 7. The cross-correlation function

According to the principle of acoustic temperature measurement, the average temperatures on different paths is obtained. The temperature measurement results of each path are shown in Table 1 . The results are close to the reference temperatures.

Table 1. Paths measurement results

\begin{tabular}{|c|c|c|c|c|c|}
\hline Path & $\begin{array}{c}\text { Average } \\
\text { tempera } \\
\text { ture } \\
\left({ }^{\circ} \mathrm{C}\right)\end{array}$ & Path & $\begin{array}{c}\text { Average } \\
\text { tempera } \\
\text { ture } \\
\left({ }^{\circ} \mathrm{C}\right)\end{array}$ & Path & $\begin{array}{c}\text { Average } \\
\text { tempera } \\
\text { ture } \\
\left({ }^{\circ} \mathrm{C}\right)\end{array}$ \\
\hline Path 1 & 721 & Path 9 & 805 & $\begin{array}{c}\text { Path } \\
17\end{array}$ & 556 \\
\hline Path 2 & 861 & $\begin{array}{c}\text { Path } \\
10\end{array}$ & 836 & $\begin{array}{c}\text { Path } \\
18\end{array}$ & 746 \\
\hline Path 3 & 741 & $\begin{array}{c}\text { Path } \\
11\end{array}$ & 680 & $\begin{array}{c}\text { Path } \\
19\end{array}$ & 734 \\
\hline Path 4 & 704 & $\begin{array}{c}\text { Path } \\
12\end{array}$ & 553 & $\begin{array}{c}\text { Path } \\
20\end{array}$ & 780 \\
\hline Path 5 & 678 & $\begin{array}{c}\text { Path } \\
13\end{array}$ & 722 & $\begin{array}{c}\text { Path } \\
21\end{array}$ & 727 \\
\hline Path 6 & 599 & $\begin{array}{c}\text { Path } \\
14\end{array}$ & 870 & $\begin{array}{c}\text { Path } \\
22\end{array}$ & 538 \\
\hline Path 7 & 800 & $\begin{array}{c}\text { Path } \\
15\end{array}$ & 789 & $\begin{array}{c}\text { Path } \\
23\end{array}$ & 805 \\
\hline Path 8 & 840 & $\begin{array}{c}\text { Path } \\
16\end{array}$ & 869 & $\begin{array}{c}\text { Path } \\
24\end{array}$ & 852 \\
\hline
\end{tabular}

\section{Conclusion}

Based on the acoustic method, this paper designs a temperature measurement system for coal-fired boilers in power plants, and can obtain the average temperature on different paths. The system uses STM32F407ZGT6 as the controller, designes the acoustic wave transmitter switching circuit, and controls the A/D converter AD7606 to collect data through the FSMC interface. In addition, the system uses the generalized crosscorrelation method PHAT-GCC to process the data. The controller uses the FPU (Floating Point Unit) and DSP instructions integrated in the controller to accelerate the operation process. According to the output signal of the acoustic wave sensor, the signal amplifying circuit and the filtering circuit are designed. The experimental results show that the design is feasible and provides reference for future research in this field.

\section{References}

1. M. Guo, Y. Yan, G. Lu, Y.H. Hu, Chinese Journal of Scientific Instrument, 39, 75 (2018).

2. G.Y. Xu, Q.Y. Xiong, R.X. Jia, R. Zhang, Application of Electronic Technique, 43, 77 (2017).

3. X.P. Sun, F. Tian, L.Y. Liu, W.J. Zhang, F.Q. Shao, Z. Xie, Instrument Technique and Sensor, 2, 26 (2005).

4. X.L. He, Power Station Auxiliary Equipment, 38, 45 (2017).

5. C. Zhu, X.X. Qu, J.Y. Lou Research. Communications Technology, 51, 1030 (2018).

6. Z.Y. Li, G.Q. Shen, Electric Safety Technology, 19, 35 (2017).

7. M. Guo, Y. Yan, Y. Hu, G. Lu, J. Zhang, Fuel, 227, 89 (2018).

8. Ł. Śladewski, K. Wojdan, K. Świrski, T. Janda, D.Nabagło, J. Chachuła, Applied Thermal Engineering, 123, 711 (2017). 